\title{
Adjunctive behavior of the rat under periodic food delivery in a 24-hour environment
}

\author{
GARY A. LUCAS, WILLIAM TIMBERLAKE, and DONALD J. GAWLEY \\ Indiana University, Bloomington, Indiana
}

\begin{abstract}
The behavior of 4 rats living in complex environments was monitored $24 \mathrm{~h}$ per day during freefeeding baseline and under conditions of periodic access to food. Under the periodic schedules, the minimum interfood interval (IFI) was increased from 16 to $512 \mathrm{sec}$ in an ascending series. Periodic food produced robust overall increases in investigation of the feeder, drinking, general activity, and rearing, but not in wheel-running. The temporal distribution of behavior within the IFI was similar across subjects and supported the hypothesis that some responses were largely time-locked to the period immediately following eating, while other responses expanded to fill the interval. However, these response differences were not adequately captured by present classification schemes. Finally, the distribution of drinking following a food pellet strongly resembled the distribution of drinking following bouts of feeding in baseline. The results suggest that adjunctive behavior stems from three sources: (1) a simple increase in the number of opportunities for expression of normal preprandial and postprandial behavior, (2) an increase in the preprandial behavior directed toward the site of expected food, and (3) an increase in the postprandial distribution of both site-directed and more general exploratory behavior. These findings suggest that adjunctive behavior is not extraneous, but is an orderly distribution of responses ordinarily related to feeding and foraging for food.
\end{abstract}

Periodic delivery of small amounts of food to a hungry organism increases a variety of responses occurring between deliveries. These responses are termed adjunctive or schedule-induced because, although related to periodic food delivery, they are not instrumental in producing food (see Falk, 1971, 1977; Roper, 1981; and Staddon, 1977, for reviews). Polydipsia (excessive drinking) is often considered the prototypical example of adjunctive behavior (Falk, 1961, 1969; Roper, 1981). However, scheduleinduced increases have been reported in several other responses, including wheel-running (King, 1974; Levitsky \& Collier, 1968), wood-chewing (Killeen, 1975; Roper, 1978), drug consumption (Lang, Latiff, McQueen, \& Singer, 1977; Oei \& Singer, 1979; Takahashi, Singer, \& Oei, 1978), and air-licking (Mendelson \& Chillag, 1970) in rats, aggression in monkeys and pigeons (Hutchinson, Azrin, \& Hunt, 1968; Yoburn \& Cohen, 1979), and pacing and cigarette smoking in humans (Wallace \& Singer, 1976).

Three major characteristics of adjunctive behavior have attracted continued interest. First, the rate or amount of behavior is often excessive compared with that occurring under schedules that do not impose delays between feeding periods. Second, the temporal distribution of responses between feeding opportunities is surprisingly consistent. And third, the emergence of adjunctive behavior cannot be explained by a simple reinforcement effect, because many adjunctive activities tend to follow rather than pre-

The present research was supported by National Institutes of Health Grant MH37892 to W.T. and G.A.L. The authors' mailing address is Department of Psychology, Indiana University, Bloomington, IN 47405. cede food delivery. Thus, the underlying cause for adjunctive behavior remains unclear. We will briefly review these characteristics of adjunctive behavior and the questions they have raised.

The issue of excessiveness has aroused considerable controversy. Roper (1981) argued that excessiveness should be evaluated against both a massed-food control (to assess the simple effects of total food intake on behavior) and an extinction control (to assess the possibility that competition from massed food may inhibit some responses). Timberlake (1982), following Falk (1971), argued that the massed-food control was the more appropriate comparison for determining excessiveness, and that extinction itself might instigate additional responding.

Whether excessiveness is a critical characterisitc of adjunctive behavior also has become an issue. Some authors have proposed a dichotomy between responses that occur in excess under periodic schedules and those that do not. In this case, the term "schedule-induced" is reserved for responses that occur in excess of baseline (e.g., Staddon, 1977). Moreover, Roper (1981) questioned the generality of schedule-induced increases in behavior, implying that few responses, other than drinking, really occur in excess under periodic schedules.

Somewhat greater agreement has been reached about the distribution and ordering of responses between food presentations, although controversy remains. Staddon (1977; see also Staddon \& Ayres, 1975; Staddon \& Simmelhag, 1971) argued for two classes of behavior following feeding, interim and facultative behavior, and one class of behavior preceding feeding, terminal behavior. Interim responses are categorized as "incentive-related", 
responses, like drinking, that emerge immediately following the incentive (food) and are likely to become excessive. According to Staddon (1977), facultative responses are "neutral" activities, like wheel-running or grooming, that serve to fill time between interim and terminal responses. Facultative responses are not predicted to occur in excess of baseline amounts. Terminal responses, like pecking in pigeons, immediately precede food delivery and most often resemble consummatory behavior.

Recently, Cohen, Looney, Campagnoni, and Lawler (1985; see also Campagnoni, Lawler, \& Cohen, 1986) argued for an alternative classification of postfeeding behavior into a time-locked repetitive class and a general activity class. They argued that behavior of the first class, represented by drinking and conspecific attack, has a more stereotyped "reflexive" quality and that its occurrence is largely limited to a fixed time after feeding. In contrast, the general-activity class is characterized by gross motor activities, is more sensitive to stimulus change, and is predicted to expand in a constant proportion to the size of the interval. In contrast to Staddon (1977), Cohen et al. (1985) argued that both classes may be expected to increase under intermittent schedules.

Attempts to provide a single causal explanation for adjunctive behavior have proven largely unsuccessful (Falk, 1977; Staddon, 1977). Most explanations have posited a special relationship between feeding and the adjunctive behavior in question, such as drinking (Kissileff, 1969) or wheel-running (e.g., Melcer \& Timberlake, 1986). Roper (1980) gave the argument an empirical form by suggesting that adjunctive behavior may show the same temporal pattern of responding that accompanies normal consummatory behavior. However, little evidence has been provided documenting the resemblance of the distributions of pre- and postprandial ${ }^{1}$ behavior that occurs under normal feeding conditions to similar distributions that occur under periodic schedules.

The present article describes the distributions of adjunctive behavior that developed in 4 rats housed in complex behavioral environments under 24 - $h$ schedules of periodic feeding. Previous work (Petersen \& Lyon, 1978) has shown that adjunctive drinking occurs readily under periodic schedules in a 24 -h environment. The present procedure began with a free-feeding baseline and then imposed a periodic feeding schedule by delaying the time between successive food pellets. The interval between pellet deliveries was extended across conditions from 16 to $512 \mathrm{sec}$. The complex environment permitted us to record simultaneously a number of potential adjunctive activities, including drinking, wheel-running, wood-block chewing, exploratory rearing, general activity, and nesting.

This approach to the study of adjunctive behavior has several advantages. First, the $24-\mathrm{h}$ schedule provided a complete and "ecologically fair" behavioral baseline. It included both periods of eating and periods of activity, both before and after feeding, that were free from competition with food (as suggested by Roper, 1981). This continuous measurement of behavior across the entire day obviated the need for special control conditions by providing a clear test of whether increased responding between food pellets occurred in excess of the daily baseline or simply resulted from the redistribution of behavior within the day.

Second, the large number of behavioral measures provided an extensive test of the classification systems proposed by Staddon (1977) and Cohen et al. (1985). Specifically, it allowed us to compare different types of behavior for excessiveness and temporal distribution within the interfood interval, and to examine the hypotheses that some types of behavior are "time-locked" with respect to feeding and that some classes of behavior will increase under periodic food presentation and others will not.

Finally, the present approach allowed us to examine whether adjunctive behavior was related to the behavior that occurred around normal feeding. First, we compared the distribution of behavior during the interfood interval of periodic feeding schedules with the distribution of behavior that occurred before and after feeding in the freefeeding baseline. Second, we attempted to relate, on the basis of its timing and organization, interfood responding to natural foraging sequences in the rat. The results suggested that adjunctive behavior was closely related both to the distribution of behavior that occurred around baseline feeding and to behavior appropriate to food-related search.

\section{METHOD}

\section{Subjects}

Four female Sprague-Dawley albino rats were obtained from the local breeding colony (Indiana University). The rats had been adapted to living in the experimental chambers for 90 days prior to the beginning of the study. They were 180 days old and weighed $300-350 \mathrm{~g}$ at the start of the present procedures.

\section{Apparatus}

The test chamber consisted of a $30 \times 30 \times 30 \mathrm{~cm}$ main compartment in which food, water, a sniff hole, and a wood block were available. (Data for sniffing are not reported here.) A diagram of the apparatus is shown in Figure 1. All locations are given with respect to the feeder wall, which was designated as the "front" wall. Both the front and back walls of the main chamber were constructed from aluminum. An Acme running wheel $(36 \mathrm{~cm}$ in diameter $\times 14 \mathrm{~cm}$ wide) was located to the left of the main chamber. A transparent Plexiglas access door formed the right side of the main compartment. A small L-shaped sheet-metal enclosure (the nest) could be entered through an opening in the left side of the back wall. The nest had a wire grid floor and was covered with a sheet-metal roof $12 \mathrm{~cm}$ above the floor.

The location of the rat was determined by weight-sensitive switches mounted in the nest and main chamber floors. Location in the main chamber was further subdivided into four quadrants (marked by dashed lines in Figure 1) by a double-tilt floor. A Plexiglas barrier extending $5 \mathrm{~cm}$ from the wall divided the feeder and watering area and ensured that the rat was required to move into the appropriate quadrant in order to feed or drink.

The bottom edge of all access areas for food, water, and the nest entry were located about $5 \mathrm{~cm}$ above the chamber floor. Two retractable levers were also set $5 \mathrm{~cm}$ above the floor on each side of the 


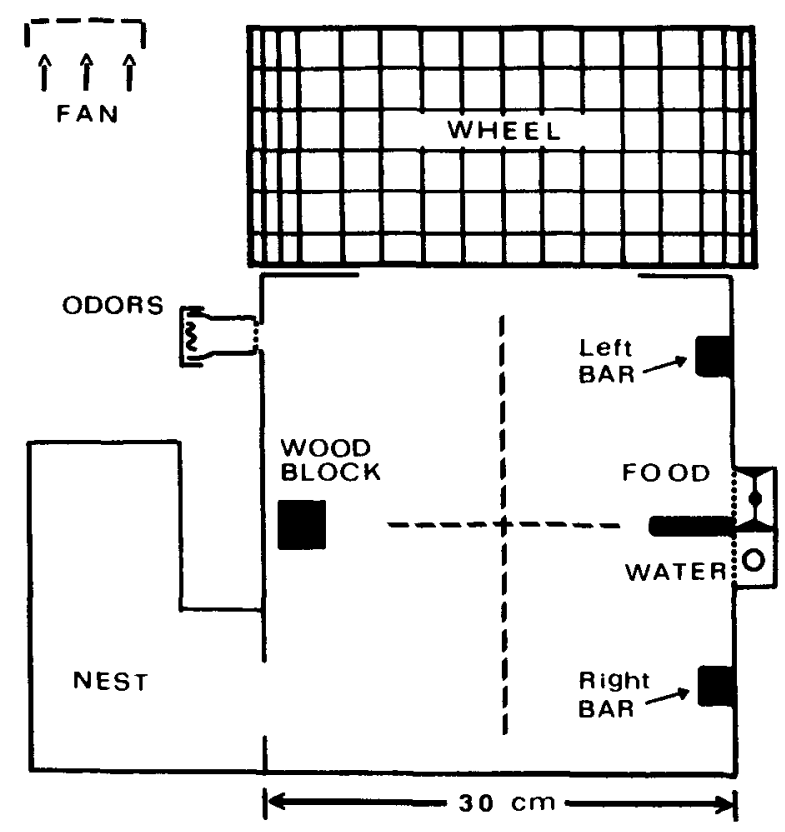

Figure 1. A diagram of the experimental chamber. The dashed lines indicate the axes of the double-tilt floor used to divide location in the main compartment into quadrants. Contact panels to measure rearing were located above the front (feeder) and back (woodblock) walls.

feeder wall. Nose entries of $0.5 \mathrm{~cm}$ or greater into the food or water magazines were determined by infrared photodetectors. Each complete rotation of the running wheel was recorded when a flag on the rim interrupted a pair of infrared photodetector elements. The order of interruption identified the direction of rotation. Rearing within the main chamber was detected by two contact-sensitive panels $(7.6 \times 30 \mathrm{~cm})$ located along the length of the front and back walls and $12.7 \mathrm{~cm}$ above the floor. A $5 \times 5 \times 3.5 \mathrm{~cm}$ wood block (untreated white pine) was located near the center of the back wall. The block was secured on a $6.4-\mathrm{cm}$-long bolt projecting upward from a spring-mounted base. The base was mounted $2.5 \mathrm{~cm}$ above the floor. Gnawing or other displacement of the block moved the bolt, which activated contact sensors located in the base.

The feeder opening $(3.2 \mathrm{~cm}$ wide $\times 5 \mathrm{~cm}$ high) was recessed $2.5 \mathrm{~cm}$ into the left side of the front wall. Food pellets (94-mg rodent pellets, Bio Serv, Frenchtown, NJ) were delivered into a $\mathrm{V}$-shaped trough at the bottom of the opening. The delivery of the pellet interrupted an infrared photodetector circuit at the bottom of the trough. The removal of a pellet (an eat) was detected when the circuit was completed. The order and timing of the pellet delivery sequence was strictly monitored in software to prevent the activity of the rat around the feeder from producing false signals.

The water opening $(3.2 \mathrm{~cm}$ wide $\times 5 \mathrm{~cm}$ high) was recessed $2.5 \mathrm{~cm}$ into the right side of the front wall. A $1.0 \mathrm{~cm}$-diam brass bowl was situated at the bottom of this opening. Drinking from the bowl was detected by means of a continuity circuit between the brass bowl and the water source that was interrupted when the bowl was emptied. Additional water was delivered in 0.05-ml units through a 2-mm opening in the bottom of the bowl via a solenoid-operated valve. Because the bowl could be refilled within $0.1 \mathrm{sec}$, this procedure provided a continuous source of water as the animal drank while intake was being measured to a $0.05-\mathrm{ml}$ resolution.

Each test chamber was enclosed in a sound-attenuating shell. The four chambers were further isolated within a dedicated temperaturecontrolled room maintained at $21^{\circ} \mathrm{C}\left( \pm 1^{\circ} \mathrm{C}\right)$ and $50 \%( \pm 10 \%)$ relative humidity. Exhaust fans were vented to the outside to isolate odor cues. A 12:12-h light:dim cycle was in effect at all times. Direct lighting of the main chamber and wheel area was provided by a 8-W fluorescent bulb located in the ceiling of the main chamber. Illuminance at the center floor level of the main chamber was $30 \mathrm{~lx}$ as measured by an Electro-Optics (Salem, MA) Model 550 radiometer/photometer. When the houselight was off, dim ambient light $(0.02 \mathrm{~lx})$ was provided through the observation window by an indirect light source located in the ceiling of the experimental room.

\section{Procedure}

Daily maintenance. Routine cleaning, replenishing of pellets, and maintenance and adjustment of equipment were performed daily. These maintenance periods were distributed irregularly during the light time (when rats showed low activity). During these periods, the rat was confined to the nest by manually closing the nest door. To minimize the effects of handling, body weight was assessed by removing the nest and weighing the rat and nest together. These maintenance periods typically took less than $10 \mathrm{~min}$ per subject. The nest doors were programmed to open $15 \mathrm{~min}$ after all maintenance had ended.

Food constraints. During the first 40 days, each subject was provided with unconstrained access to food. This was achieved by delivering a new food pellet as soon as the previous pellet was removed. Baseline measures of feeding and interfeeding distributions were based on the last 6 days of this free-feeding baseline (Base1).

Following establishment of baseline, access to food was restricted by imposing an ascending series of delays betwen the time a pellet was removed from the feeder and the time it was replaced. The interfood intervals (IFIs) were 16, 32, 64, 128, 256, and 512 sec. Each condition was in effect for 12 successive days, except for the IFIs 128 and 512 , which were in effect for 18 and 14 days, respectively. Subject S3 lost weight rapidly under the IFI 512 condition and was supplemented with additional food in the nest after 6 days on this condition. The data reported for this subject were therefore restricted to the first 6 days in this condition. Means for all other conditions were based on the last 6 days. Following IFI 512, each subject was returned to unconstrained-access baseline (Base2) for $\mathbf{4 0}$ days.

\section{Experimental Control and Data Analysis}

Control of each chamber was arranged by an IBM personal computer and locally constructed solid state interface located in a separate room. All contingencies and timing were managed by Spyder Systems' Conman software (Bloomington, IN) operating at 0.1-sec resolution. A 32-channel event record was synthesized by the software for each chamber and was stepped each $4 \mathrm{sec}$. The presence or absence of each behavior was continuously monitored and written to the event record providing a continuous measure of the occurrence of each behavior at a 4-sec resolution. These records provided the source for all measures of time spent in each activity. In addition, absolute counts of discrete events, such as pellets, 0.05-ml drinks, and wheel turns, were recorded at 30 -min intervals.

A brief description of the behavior categories used in the study is given in Table 1 . Two of the categories described in Table 1 require some additional comment. The categories "wait by feeder" and "wait by water" were defined as time spent in the quadrant of the main chamber near either the food or water that could not be accounted for on the basis of time spent eating (drinking), looking in the feeder (waterer), rearing, or other movements around the respective areas. Numerous observations of the rats during daytime feeding and drinking episodes indicated that these periods mainly included time spent sniffing along the floor, grooming, and periods of inactivity that seemed appropriately labeled "waiting." In baseline, the greatest proportion of these activities occurred after eating and drinking. However, it was not unusual for a rat to approach the feeder or water area and pause briefly before eating.

A log-survivor analysis (Fagan \& Young, 1978; Lehner, 1979) of baseline intereating intervals (not shown) indicated that a continuous bout of feeding could be characterized by intereating inter- 
Table 1

Description of Behavior Categories

\begin{tabular}{ll}
\hline \multicolumn{1}{c}{ Behavior } & \multicolumn{1}{c}{ Description } \\
Eat & Food pellet ( $94 \mathrm{mg}$ ) removed from feeder. \\
Wait by Feeder & $\begin{array}{l}\text { Time spent in the left-front quadrant of the main } \\
\text { chamber in excess of time spent with head in feeder, } \\
\text { rearing, pressing the bar, or moving to another lo- } \\
\text { cation. Common behaviors seen in this category were } \\
\text { sniffing along the floor, grooming, and periods of } \\
\text { inactivity (waiting). }\end{array}$
\end{tabular}

Drink Consumption of 0.05 -ml volume of water.

Head in Water Head enters at least $0.5 \mathrm{~cm}$ inside water magazine.

Wait by Water Time spent in the right-front quadrant of the main chamber in excess of time spent with head in water, rearing, pressing the bar, or moving to another location (see wait by feeder).

Activity Movement across the main compartment of the chamber.

Chew Any movement of the wood block sufficient to displace it by $0.5 \mathrm{~cm}$.

Nest Located in nest enclosure.

Rear Touching the contact sensitive panels along the top of the front or back walls while standing on the floor.

Run A complete revolution of the wheel in a clockwise or counter-clockwise direction.

vals of $32 \mathrm{sec}$ or less (mode 8-12 sec). That is, there was a distinct change in the probability of eating another pellet after pauses of $32 \mathrm{sec}$ or longer. Therefore, subjects were considered to be continuing in the same bout of feeding as long as they ate pellets within $32 \mathrm{sec}$ of delivery. A similar analysis under periodic pellet delivery indicated that this same criterion, responsiveness to food within $32 \mathrm{sec}$, could be used to define an "extended bout" when the time between pellets was delayed. On this basis, subjects were considered to be continuing in an extended feeding bout as long as they ate each pellet within $32 \mathrm{sec}$ after it was delivered. All behavior that occurred between pellets that were consumed within $32 \mathrm{sec}$ of delivery was therefore classified as occurring within an extended feeding bout. Behavior that occurred at all other times was classified as occurring between bouts.

\section{RESULTS}

\section{Excessiveness}

Table 2 shows the mean daily body weight and response totals in the first baseline (Base1) for each subject. All measures are reported as the total number of 4-sec bins in which each behavior was recorded (except body weight, which is given in grams). The data in all subsequent conditions are presented as percentages of the first baseline scores. Because it required more than $4 \mathrm{sec}$ for a rat to consume a $94-\mathrm{mg}$ food pellet, the total number of 4-sec bins in which an eat was recorded equals the mean number of pellets eaten per day. In comparison, the baseline score for drinks represents consumption of about 2 drinks $(0.1 \mathrm{ml})$ per $4-\mathrm{sec}$ period. The mean individual intake rates per 4-sec interval of drinking in baseline were 0.097 , $0.088,0.116$, and $0.114 \mathrm{ml}$ for Subjects S1 to S4, respec- tively, and these drinking rates remained relatively constant across all conditions.

Inspection of Table 2 showed robust schedule-induced increases for head in feeder, wait by feeder, drink, head in water, wait by water, general activity, and rear. Occasional increases were also seen in wood-block chewing by 3 subjects and running by 2 subjects, but these effects were clearly less extensive and reliable. In fact, for Subject S1, running actually decreased as the IFI increased. Time spent in the nest showed a modest increase for 2 subjects under the initial constraints but decreased under the longer constraints, and eating decreased reliably with increased IFI. Although the reduction in food intake resulted in a substantial weight loss for all subjects by the IFI 512, there is little indication that a reduction in body weight was necessary to produce increases in adjunctive behavior. For all subjects, increases in many responses preceded any marked decrease in weight.

An examination of event records for individual subjects showed several changes in the way behavior was distributed under periodic feeding. At IFIs 16 and 32, the rats' feeding bouts continued to occur as continuous blocks of feeder-directed activity distinctly separated from periods of such other behavior as drinking and running. However, under IFI 64, other responses began to appear within the intervals of ongoing feeding bouts (see Figures 2 and 3). In other words, the animal began to express other responses on a time-sharing basis with eating (McFarland, 1974), regularly interrupting these responses when food was imminent. At IFIs 128, 256, and 512, the majority of all responses, except nest time, occurred within the IFI. This time-sharing strategy appeared to be necessary, in part, because the rats continued to spend a substantial amount of time within the nest enclosure. Note that even under the most severe constraints over $16 \mathrm{~h}$ per day was spent within the nest.

\section{IFI Patterning and Time-Locked Responses}

The distribution of behavior within the IFI is shown in Figures 2 and 3 for the four longest interfood intervals across 4-sec bins. The measure used was the percentage of total intervals in which each behavior was recorded. To accommodate the wide range of scores, the data are presented as the log of this percentage. Accordingly, a $\log$ of 1.0 indicates that a response occurred in $10 \%$ of the possible intervals, a log of 2.0 indicates that a response occurred in $100 \%$ of the intervals.

Although individual differences are apparent, the responses displayed in the left column under each condition reveal a systematic pattern of postpellet activity emerging first in continued exploration of the feeder (head in feeder), followed by time spent near the feeder (wait by feeder), followed by a transition to drinking and wait by water. As previously noted, the wait-by-food category included episodes of face grooming and episodes of sniffing along the floor area beneath the feeder. The wait-bywater category also included these responses, although 
Table 2

Mean Baseline 1 Totals and the Percent of Baseline 1 Across Conditions

\begin{tabular}{|c|c|c|c|c|c|c|c|c|c|}
\hline & \multirow[b]{2}{*}{ Subject } & \multirow[b]{2}{*}{ Base 1 } & \multicolumn{7}{|c|}{ Interfood Intervals } \\
\hline & & & 16 & 32 & 64 & 128 & 256 & 512 & Base2 \\
\hline Body Weight & $\begin{array}{l}1 \\
2 \\
3 \\
4\end{array}$ & $\begin{array}{l}354.8 \\
350.7 \\
353.0 \\
316.7\end{array}$ & $\begin{array}{c}98.5 \\
102.6^{*} \\
101.1 \\
101.0\end{array}$ & $\begin{array}{c}98.8 \\
99.3 \\
104.2^{*} \\
100.6\end{array}$ & $\begin{array}{l}98.0 \dagger \\
101.5 \\
105.9^{*} \\
100.1\end{array}$ & $\begin{array}{r}97.7 \dagger \\
104.1^{*} \\
97.5 \dagger \\
101.3\end{array}$ & $\begin{array}{l}93.7 \dagger \\
99.8 \\
89.0 \dagger \\
98.1 \dagger\end{array}$ & $\begin{array}{l}85.1 \dagger \\
92.2 \dagger \\
84.1 \dagger \\
85.6 \dagger\end{array}$ & $\begin{array}{l}106.0^{*} \\
111.8^{*} \\
102.8^{*} \\
105.8^{*}\end{array}$ \\
\hline Eat & $\begin{array}{l}1 \\
2 \\
3 \\
4\end{array}$ & $\begin{array}{l}193.5 \\
174.4 \\
223.5 \\
174.1\end{array}$ & $\begin{array}{l}73.6 \dagger \\
82.9 \dagger \\
91.8 \\
97.5\end{array}$ & $\begin{array}{l}76.6 \dagger \\
73.9 \dagger \\
93.3 \\
96.5 \dagger\end{array}$ & $\begin{array}{c}71.0 \dagger \\
85.6 \dagger \\
85.2 \dagger \\
102.4\end{array}$ & $\begin{array}{l}80.5 \dagger \\
86.8 \dagger \\
66.7 \dagger \\
84.1 \dagger\end{array}$ & $\begin{array}{l}63.9 \dagger \\
66.5 \dagger \\
51.2 \dagger \\
92.3 \dagger\end{array}$ & $\begin{array}{l}48.5 \dagger \\
50.7 \dagger \\
29.8 \dagger \\
51.3 \dagger\end{array}$ & $\begin{array}{c}97.4 \\
113.4^{*} \\
100.5 \\
108.8^{*}\end{array}$ \\
\hline Head in Feeder & $\begin{array}{l}1 \\
2 \\
3 \\
4\end{array}$ & $\begin{array}{l}360.7 \\
462.1 \\
315.3 \\
337.4\end{array}$ & $\begin{array}{l}135.4^{*} \\
123.2^{*} \\
156.6^{*} \\
159.7^{*}\end{array}$ & $\begin{array}{l}125.4^{*} \\
157.1^{*} \\
195.0^{*} \\
135.1^{*}\end{array}$ & $\begin{array}{l}108.4^{*} \\
145.4^{*} \\
189.1^{*} \\
165.2^{*}\end{array}$ & $\begin{array}{l}134.1^{*} \\
216.6^{*} \\
244.1^{*} \\
155.8^{*}\end{array}$ & $\begin{array}{l}188.9^{*} \\
384.9^{*} \\
297.6^{*} \\
564.6^{*}\end{array}$ & $\begin{array}{l}101.0 \\
372.2^{*} \\
241.2^{*} \\
256.7^{*}\end{array}$ & $\begin{array}{r}97.0 \\
96.9 \\
101.4 \\
107.0\end{array}$ \\
\hline Wait by Feeder & $\begin{array}{l}1 \\
2 \\
3 \\
4\end{array}$ & $\begin{array}{r}96.0 \\
99.0 \\
198.0 \\
132.0\end{array}$ & $\begin{array}{r}143.7 \\
109.1 \\
65.2 \\
65.9\end{array}$ & $\begin{array}{l}779.7^{*} \\
480.3^{*} \\
198.5^{*} \\
378.4^{*}\end{array}$ & $\begin{array}{r}1190.6^{*} \\
713.6^{*} \\
187.9^{*} \\
565.9^{*}\end{array}$ & $\begin{array}{c}1904.7^{*} \\
830.3^{*} \\
115.9 \\
722.7^{*}\end{array}$ & $\begin{array}{c}2048.4^{*} \\
119.7 \\
231.1^{*} \\
1247.7^{*}\end{array}$ & $\begin{array}{c}2009.4^{*} \\
142.4 \\
103.8 \\
825.0^{*}\end{array}$ & $\begin{array}{c}162.5^{*} \\
130.3 \\
95.5 \\
105.7\end{array}$ \\
\hline Drink & $\begin{array}{l}1 \\
2 \\
3 \\
4\end{array}$ & $\begin{array}{l}165.9 \\
259.3 \\
190.1 \\
304.0\end{array}$ & $\begin{array}{c}109.4 \\
80.3 \dagger \\
106.5 \\
110.0^{*}\end{array}$ & $\begin{array}{c}107.0 \\
94.5 \\
153.7^{*} \\
116.8^{*}\end{array}$ & $\begin{array}{c}123.0^{*} \\
98.3 \\
158.5^{*} \\
103.8\end{array}$ & $\begin{array}{l}229.5^{*} \\
197.2^{*} \\
120.0^{*} \\
105.1\end{array}$ & $\begin{array}{l}203.0^{*} \\
306.5^{*} \\
150.5^{*} \\
187.0^{*}\end{array}$ & $\begin{array}{r}206.1^{*} \\
215.7^{*} \\
148.5^{*} \\
81.9 \dagger\end{array}$ & $\begin{array}{c}139.4 * \\
90.6 \\
94.8 \\
89.2\end{array}$ \\
\hline Head in Water & $\begin{array}{l}1 \\
2 \\
3 \\
4\end{array}$ & $\begin{array}{l}217.6 \\
280.2 \\
234.6 \\
370.6\end{array}$ & $\begin{array}{c}103.4 \\
83.8 \dagger \\
108.8 \\
111.9\end{array}$ & $\begin{array}{c}113.6^{*} \\
95.8 \\
158.6^{*} \\
118.6^{*}\end{array}$ & $\begin{array}{l}121.0^{*} \\
102.1 \\
174.0^{*} \\
101.3\end{array}$ & $\begin{array}{l}214.8^{*} \\
203.5^{*} \\
115.7^{*} \\
112.0\end{array}$ & $\begin{array}{l}204.4^{*} \\
316.0^{*} \\
164.1^{*} \\
201.8^{*}\end{array}$ & $\begin{array}{c}215.6^{*} \\
222.2^{*} \\
175.2^{*} \\
99.4\end{array}$ & $\begin{array}{l}128.9 * \\
92.2 \\
93.7 \\
85.0 \dagger\end{array}$ \\
\hline Wait by Water & $\begin{array}{l}1 \\
2 \\
3 \\
4\end{array}$ & $\begin{array}{l}39.6 \\
61.5 \\
45.8 \\
91.5\end{array}$ & $\begin{array}{c}157.5 \\
75.7 \\
33.0 \dagger \\
142.0\end{array}$ & $\begin{array}{c}227.0^{*} \\
91.9 \\
60.3 \\
247.1^{*}\end{array}$ & $\begin{array}{l}153.5 \\
140.5 \\
663.9^{*} \\
223.4^{*}\end{array}$ & $\begin{array}{l}776.8^{*} \\
235.1^{*} \\
153.5 \\
246.8^{*}\end{array}$ & $\begin{array}{l}496.4^{*} \\
445.9^{*} \\
133.0 \\
564.6^{*}\end{array}$ & $\begin{array}{c}465.1^{*} \\
129.7 \\
56.7 \\
296.2^{*}\end{array}$ & $\begin{array}{r}115.2 \\
56.8 \\
153.1 \\
149.2\end{array}$ \\
\hline Activity & $\begin{array}{l}1 \\
2 \\
3 \\
4\end{array}$ & $\begin{array}{l}204.9 \\
136.6 \\
378.7 \\
283.8\end{array}$ & $\begin{array}{c}93.6 \\
118.7 \\
83.0 \dagger \\
105.4\end{array}$ & $\begin{array}{r}99.9 \\
142.7^{*} \\
83.2 \dagger \\
122.6^{*}\end{array}$ & $\begin{array}{l}106.6 \\
180.8^{*} \\
162.3^{*} \\
139.0^{*}\end{array}$ & $\begin{array}{l}160.2^{*} \\
228.6^{*} \\
136.5^{*} \\
153.3^{*}\end{array}$ & $\begin{array}{l}287.9^{*} \\
294.3^{*} \\
169.9^{*} \\
244.0^{*}\end{array}$ & $\begin{array}{l}511.2^{*} \\
411.3^{*} \\
194.5^{*} \\
210.2^{*}\end{array}$ & $\begin{array}{c}103.0 \\
78.5 \dagger \\
47.2 \dagger \\
78.2 \dagger\end{array}$ \\
\hline Chew & $\begin{array}{l}1 \\
2 \\
3 \\
4\end{array}$ & $\begin{array}{r}12.0 \\
7.7 \\
10.5 \\
10.8\end{array}$ & $\begin{array}{c}22.5 \dagger \\
49.0 \\
115.0 \\
115.3\end{array}$ & $\begin{array}{r}90.0 \\
29.4 \dagger \\
610.0^{*} \\
15.3 \dagger\end{array}$ & $\begin{array}{c}23.8 \dagger \\
25.5 \dagger \\
178.5 \\
79.2\end{array}$ & $\begin{array}{c}88.7 \\
76.5 \\
720.0^{*} \\
445.8^{*}\end{array}$ & $\begin{array}{c}52.5 \\
423.5^{*} \\
861.4^{*} \\
104.2\end{array}$ & $\begin{array}{c}92.5 \\
186.3^{*} \\
917.0^{*} \\
84.7\end{array}$ & $\begin{array}{c}66.3 \\
58.8 \\
128.0 \\
304.2^{*}\end{array}$ \\
\hline Nest & $\begin{array}{l}1 \\
2 \\
3 \\
4\end{array}$ & $\begin{array}{l}17042.8 \\
18772.1 \\
17391.9 \\
16509.2\end{array}$ & $\begin{array}{c}106.7^{*} \\
100.4 \\
114.6^{*} \\
96.8\end{array}$ & $\begin{array}{c}98.6 \\
96.5 \dagger \\
103.5 * \\
95.0\end{array}$ & $\begin{array}{c}107.4^{*} \\
95.6 \dagger \\
99.0 \\
94.3\end{array}$ & $\begin{array}{l}95.5 \dagger \\
88.9 \dagger \\
96.4 \\
93.1\end{array}$ & $\begin{array}{l}92.6 \dagger \\
89.6 \dagger \\
91.5 \dagger \\
79.0 \dagger\end{array}$ & $\begin{array}{l}89.3 \dagger \\
89.8 \dagger \\
92.9 \dagger \\
85.4 \dagger\end{array}$ & $\begin{array}{c}101.5 \\
97.8 \\
107.0^{*} \\
98.0\end{array}$ \\
\hline Rear & $\begin{array}{l}1 \\
2 \\
3 \\
4\end{array}$ & $\begin{array}{l}39.3 \\
31.2 \\
40.5 \\
58.8\end{array}$ & $\begin{array}{c}85.1 \\
119.7 \\
85.9 \\
117.3^{*}\end{array}$ & $\begin{array}{c}112.2 \\
120.7 \\
52.2 \dagger \\
116.6^{*}\end{array}$ & $\begin{array}{c}87.8 \\
215.4^{*} \\
111.9 \\
130.6^{*}\end{array}$ & $\begin{array}{l}141.6^{*} \\
285.1^{*} \\
163.0^{*} \\
148.5^{*}\end{array}$ & $\begin{array}{l}202.3^{*} \\
190.9^{*} \\
157.0^{*} \\
191.3^{*}\end{array}$ & $\begin{array}{l}243.1^{*} \\
206.7^{*} \\
126.7^{*} \\
192.3^{*}\end{array}$ & $\begin{array}{l}133.6^{*} \\
102.4 \\
107.4 \\
133.2^{*}\end{array}$ \\
\hline Run & $\begin{array}{l}1 \\
2 \\
3 \\
4\end{array}$ & $\begin{array}{r}455.0 \\
88.5 \\
947.8 \\
506.0\end{array}$ & $\begin{array}{c}31.6 \dagger \\
133.9 \\
73.7 \\
106.8\end{array}$ & $\begin{array}{c}70.8 \dagger \\
125.8 \\
31.6 \dagger \\
111.6\end{array}$ & $\begin{array}{c}37.9 \dagger \\
174.7^{*} \\
34.0 \dagger \\
122.5\end{array}$ & $\begin{array}{c}57.8 \dagger \\
169.5^{*} \\
84.5 \\
92.9\end{array}$ & $\begin{array}{c}58.5 \dagger \\
98.0 \\
84.1 \\
124.8\end{array}$ & $\begin{array}{c}84.4 \\
140.7 \\
103.8 \\
250.0^{*}\end{array}$ & $\begin{array}{l}54.0 \dagger \\
47.1 \dagger \\
52.1 \dagger \\
45.0 \dagger\end{array}$ \\
\hline
\end{tabular}

Note-Except for body weight, which is reported in grams, first baseline (Basel) data are reported as the number of 4 -sec bins $\{21,600$ bins were scored per day $)$ in which the behavior occurred. Data in all subsequent conditions are reported as percentages of the first baseline. (Base $2=$ second baseline; see Table 1 for a description of the behavior categories.) *Greater than two standard errors above the first baseline. $\dagger$ Greater than two standard errors below the first baseline.

it was our impression that this category typically included less floor sniffing and more extensive body grooming than seen after feeding.

Activity and rearing also increased soon after pellet delivery. Note that the activity measure was based on changes in location within the chamber. These changes in location were evident across the entire interval. The peak of activity after a pellet was taken resulted from a rat's tendency to grab a pellet in its mouth and move to a new location before eating. Such behavior resembled that exhibited by animals when protecting food items from theft in social feeding situations. Casual observation in- 
INTERFOOD 64 SEC
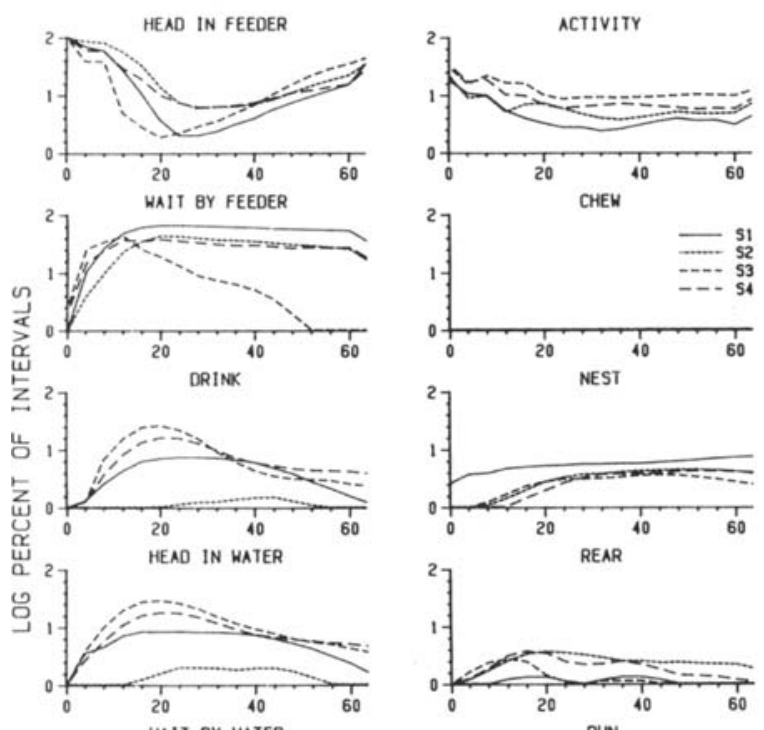

WAIT BY HATER
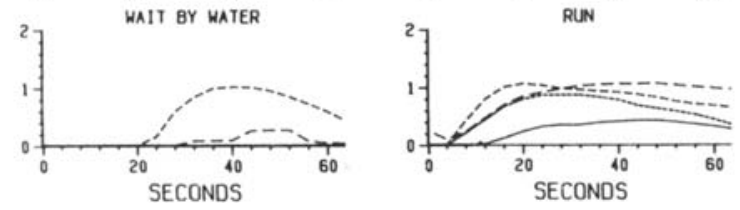

INTERFOOD 128 SEC
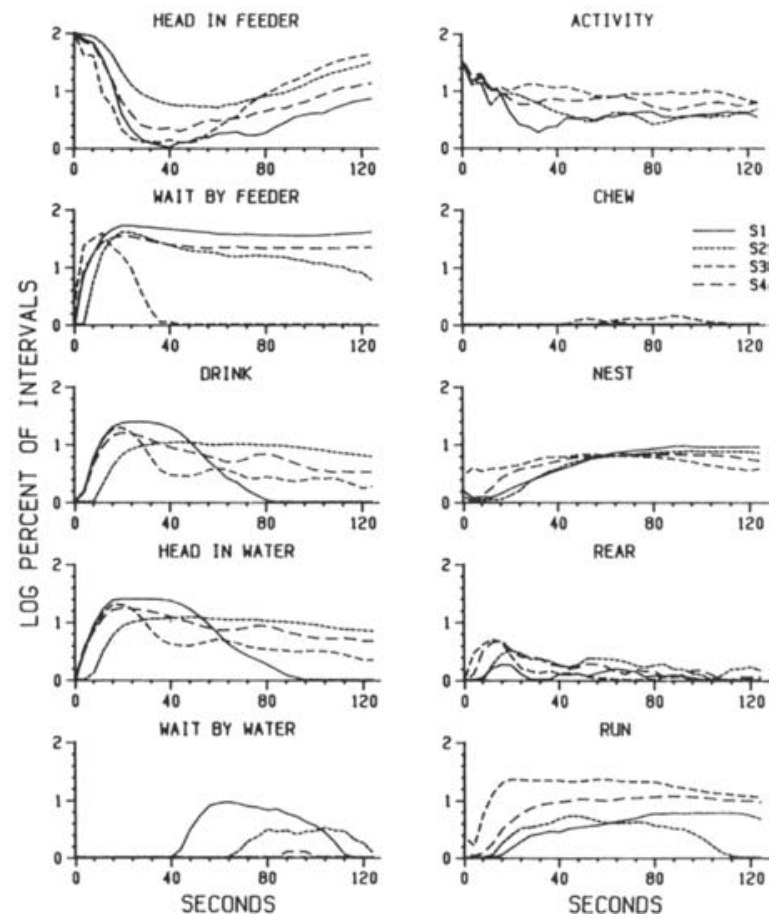

Figure 2. The distribution of responses in 4-sec bins following eating $(0 \mathrm{sec})$ when the minimum IFI was 64 or 128 sec. The data for each subject are plotted as the $\log$ of the mean percentage of total observation bins in which each response occurred.

dicated that this "snatch and run" pattern was most likely at the start of a feeding period or during daylight feeding. The periods of rearing, although less distinct, corresponded closely in time to the periods of transition away from the food area. The remaining behavior categorieschew, nest, and run-tended to occur later in the interfood interval.

The concept of time-locked categories of behavior (Cohen et al., 1985) suggests that the processes underlying the expression of some behaviors following eating may be linked to a relatively rigid time frame. Although the mean of a response distribution may vary with the time available, the modal time of expression should reveal the presence of any dominant underlying time frame. To examine the tendency for different responses to emerge at fixed times after feeding, we determined the time from eating to the peak (mode) of each behavioral distribution and averaged these times across subjects to obtain an estimate of the dominant time of emergence for each response following eating. These "peak points" are plotted in Figure 4 across IFIs.

The plot reveals two distinct sets of behavior. One set, including wait by water, nest, run, and chew showed a peak of activity near the middle of each IFI. The absolute time of this peak increased across conditions as the IFI increased. A second set of responses composed of rear, drink, wait by feeder, and activity peaked at about the same time in each condition, although this generalization broke down at the IFI 512 condition for drink and rear. Closer inspection of this second set of responses revealed a consistent ordering with activity peaking first, followed by wait by feeder, and drinking. The ordering of the rear category appeared to move somewhat, but was generally in the time range of wait by feeder and drinking.

Although head in feeder was not included in this plot, it should be clear from Figures 2 and 3 that there were two distinct peaks of head-in-feeder activity, one postpellet peak immediately after eating and a second, preprandial peak occurring later in the interval. Note also that activity (movement) may actually be composed of two sets of behavior. In addition to the distinct time-locked postpellet bursts of movement, a second, rather flat interpellet distribution of activity was apparent at longer IFIs.

\section{Relationship to Free-Feeding Distribution}

One of our primary purposes was to compare the distribution of adjunctive behavior under periodic feeding schedules with the distributions of pre- and postprandial behavior found around unconstrained feeding periods in baseline. The comparisons for postprandial behavior were made by superimposing responding from the first (postpellet) half of the schedule distributions on the temporal distribution of each response following baseline feeding bouts. The comparisons for preprandial behavior were made by superimposing responding from the second (prepellet) half of the schedule distribution on the temporal distribution of each response preceding baseline feeding bouts. The results of these comparisons are shown in Fig- 
INTERFOOD 256 SEC

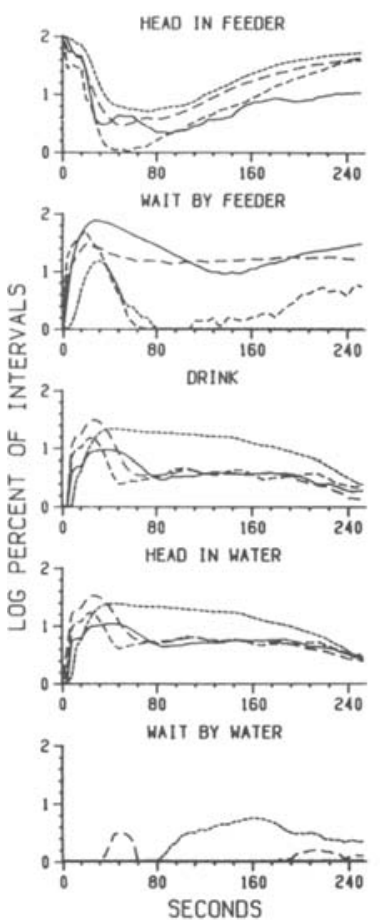

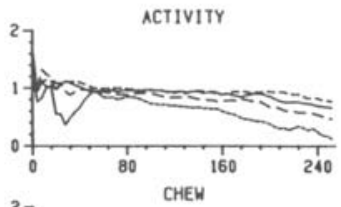
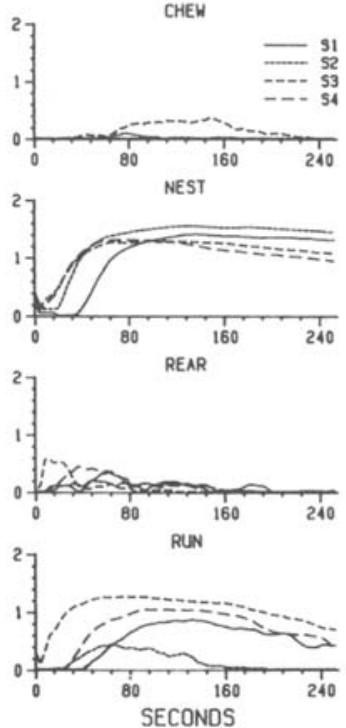

INTERFOOD 512 SEC
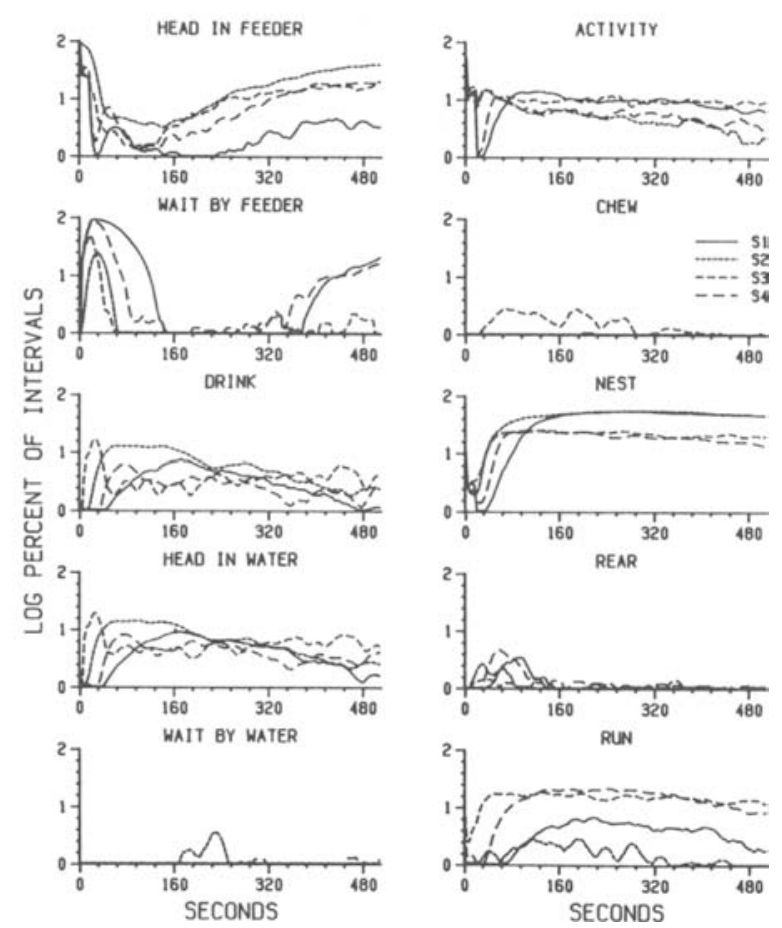

Figure 3. The distribution of responses in 4-sec bins following eating $(0 \mathrm{sec})$ when the minimum interfood interval was 256 or 512 sec. The data for each subject are plotted as the log of the mean percentage of total observation bins in which each response occurred.

ure 5 as group means plotted by $\log$ percent of each 4 -sec observation period in which each behavior was recorded. Because some measures, in particular wheel-running, did not recover baseline totals in the second baseline, the baseline data are combined averages across the last 6 days of both the first and second baseline conditions.

For statistical purposes, the distributions in Figure 5 were summarized as the mean percentage of occurrence across each interval and compared with the baseline means for the same interval using a repeated measures ANOVA. We used the mean of the distributions here because the distributions were, in most cases, relatively similar in shape across intervals, and the combination of comparisons across conditions for the mean is both convenient and powerful. The comparison of interest was whether the postprandial (or preprandial) distribution differed from the baseline distribution. The level of significance for all comparisons was set at $p<.05(d f=1,3$ for the comparison across conditions, $d f=5,15$ for the interaction).

The results of this analysis for the postprandial distribution revealed a significant increase in activity, a reduction in visits to the nest in the first $64 \mathrm{sec}$ after eating, and an increase in wait by feeder during the first $64 \mathrm{sec}$ after eating. The increases in running and rearing during this time period approached significance $(p s<.07)$. The distributions for head in feeder, head in water, and drinking during the postprandial interval did not differ significantly from the baseline distribution $(p s>.30)$.
The comparison for the preprandial interval revealed a significant increase for head in feeder at the longer intervals, and a significant increase in wait by feeder. Activity (change in location) was reduced in the $64 \mathrm{sec}$ before feeding (primarily due to the increase in waiting near the feeder). The probability of being in the nest before eating was lower at the shorter intervals but approached the baseline distribution at the longer intervals. The distribution of running in the preprandial intervals varied

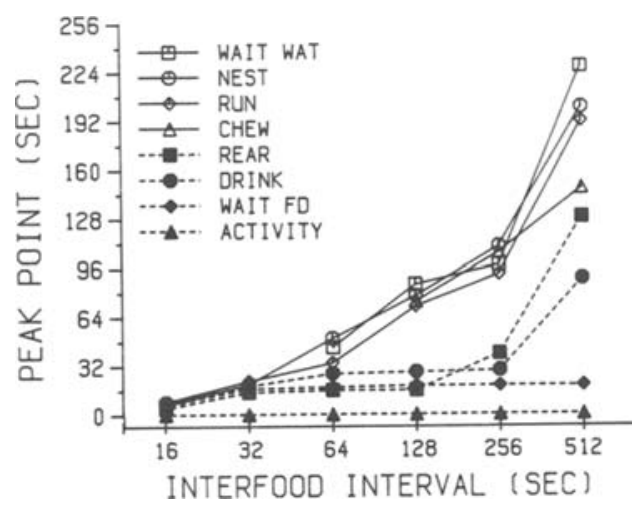

Figure 4. The mean peak point for the distributions of different responses under the periodic food schedules. Note that the measures for activity, wait by food, drinking, and rearing show a tendency to peak at about the same time after eating across most of the range of interfood intervals. 

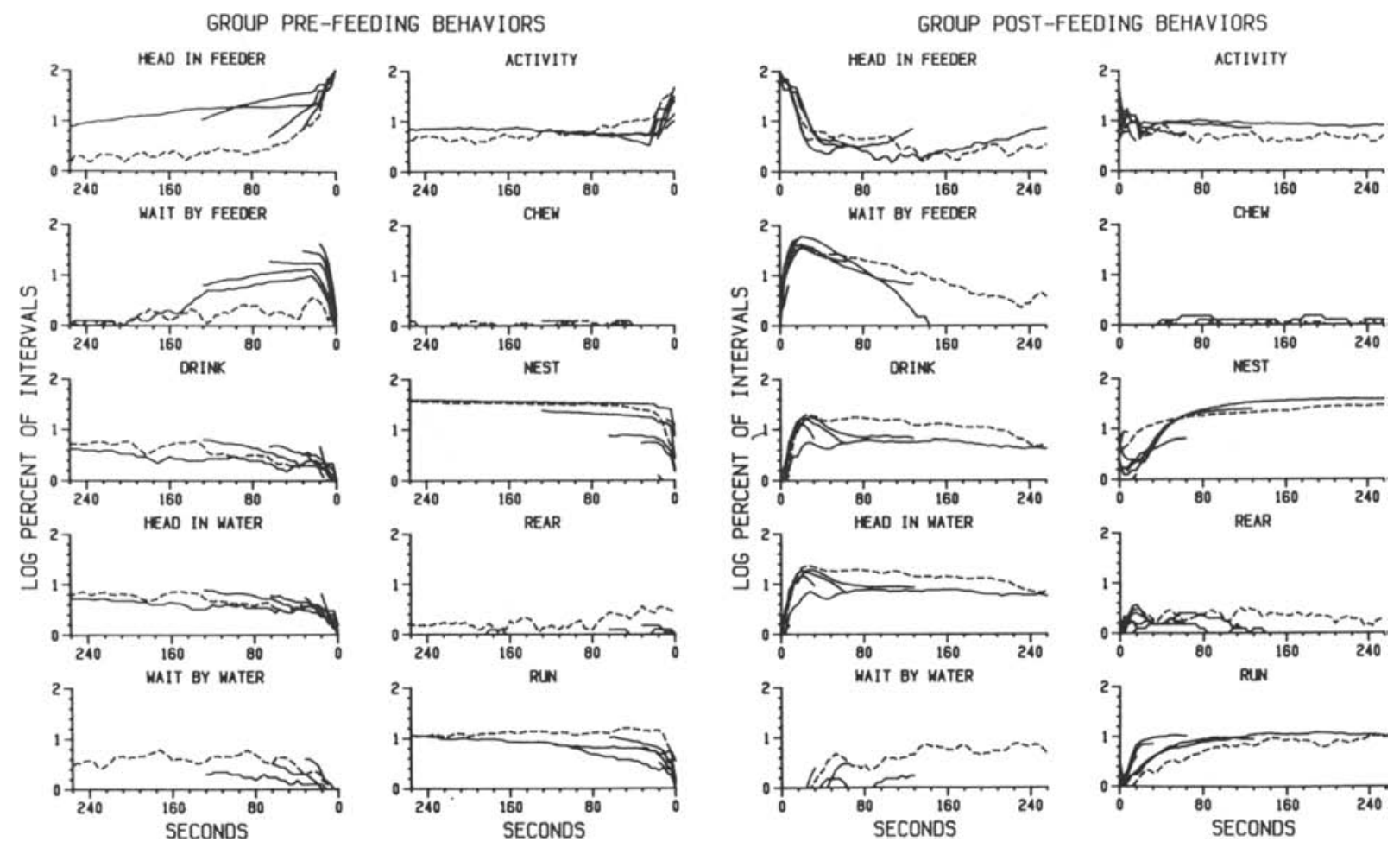

Figure 5. The distribution of responses in $4-\mathrm{sec}$ bins for the half of the interfood interval following (post-) or preceding (pre-) eating $(0 \mathrm{sec})$. The solid lines show the distributions under the delayed food conditions; the dashed line shows the distribution in free-feeding baseline across the same time scale. The data are group averages plotted as the log of the mean percentage of total observation bins in which each response occurred.

widely across subjects but did not differ significantly from baseline despite the apparent reduction seen in Figure 5. As was the case in the postprandial analysis, the preprandial distributions of head in water and drinking did not differ from baseline.

Although the mean drinks per opportunity for postprandial drinking did not differ significantly from baseline, it was our impression that the shape of the drinking distribution changed somewhat at the longest interfood intervals. To assess this possibility, we compared the schedule drinking distribution with the corresponding baseline drinking distribution using a chi-square analysis. The data for this comparison were the untransformed scores for each individual subject across 4-sec bins for the pre- and postprandial distributions. Individual chisquare values were then combined across subjects to yield a summary standard score for each schedule, taking into account the direction of change (Cochran, 1954).

The only significant effects shown by the results of the chi-square comparisons were reductions in the postprandial distributions for the IFI 256 and 512 conditions ( $z s=-2.72$ and -2.86 , respectively, both $p s<.01$ ). Inspection of individual distributions indicated that the reduction for the IFI 256 condition was almost exclusively limited to the longer postfood times. For the IFI 512 condition, 2 subjects, $\mathrm{S} 3$ and $\mathrm{S} 4$, showed consistent decreases across most of the postprandial distribution. Similar comparisons across the preprandial distributions under the schedules showed no significant differences from the baseline preprandial distribution (the absolute value of all $z s<1.1$, all $p s>.27$ ).

Because the number of IFIs varied across conditions, the data in Figure 5 do not directly correspond to the total amount of behavior that occurred under each condition, but rather show the percentage of time that the behavior occurred per feeding opportunity. This distinction is important because, to the extent that the postprandial (or preprandial) probability of occurrence remains constant across conditions, a change in total amount of behavior per day can be attributed simply to the increased opportunities for the postprandial (or preprandial) distribution to occur. This is exactly the case for drinking. Thus, it appears that the excessive drinking obtained at longer IFIs can be attributed to an increase in the frequency and duration of postprandial periods rather than to an increase in the amount of drinking per opportunity. In fact, excessive drinking occurred in the IFI 256 and 512 conditions even though the postprandial distribution of drinking was significantly lower than the baseline per opportunity distribution.

\section{DISCUSSION}

\section{Excessiveness}

The present results showed that increases in a variety of adjunctive activities were generated by periodic food 
schedules imposed over the entire 24-h day. Compared with the free-feeding condition, all subjects showed significant increases for head in feeder, wait by feeder, head in water, drink, wait by water, activity, and rearing. Thus, across a 24-h period, schedule-induced increases in behavior appear to be more robust and widespread than suggested by Roper (1981). However, not all responses increased under periodic schedules. In particular, changes in wood-chewing and wheel-running were not reliable across animals and conditions, and time spent in the nest decreased at longer intervals.

While the restrictions on access to food in the present study lead to a significant loss in body weight under the more severe constraints, increases in adjunctive behavior did not appear to depend on a loss in body weight. These results thus support the conclusion of Roper and Nieto (1979) that severe deprivation is not necessary to induce adjunctive behavior. However, other work suggests that some responses, in particular, wood-chewing (Roper \& Crossland, 1982), may show more robust changes under severe food deprivation. Such a trend for chewing was apparent in our data for 1 subject.

Although overall activity under periodic feeding increased for all subjects, total wheel-running did not increase reliably above baseline. These findings thus replicate those of Staddon and Ayres (1975), but differ from the marked increases in running under periodic feeding shown in the data of Bolles and Moot (1973), Levitsky and Collier (1968), and Melcer and Timberlake (1986). All these studies are generally consistent with Staddon's (1977) conclusion that the amount of schedule-induced running that is engendered by a situation is inversely related to the amount of space available to the rat outside the wheel (cf. Wetherington, Brownstein, \& Shull, 1977).

In addition to changes in the total amounts of many responses, the periodic presentation of food resulted in a major reorganization of behavior around feeding periods. Beginning with the 64-sec IFI, responses that occurred outside the feeding bout during baseline increasingly occurred within the IFI. McFarland (1974; see also McFarland \& Lloyd, 1973) used the term time-sharing to describe behavior relegated to pauses in the pattern of a more dominant behavior. Though the pauses in eating in the present case were forced by the schedule, it seems clear that patterns of behavior within the interfood interval were sequenced around feeding as the dominant behavior. Several characteristics of this time-sharing of responses with feeding are worth noting. First, the increases in daily response totals that developed for some behaviors under periodic feeding coincided with the tendency for those behaviors to be time-shared with feeding. Second, except perhaps at the 512-sec interval, this reorganization was not forced by a lack of time to both eat and perform all other responses independently at baseline levels.

A surprising aspect of these data was the large amount of time the rats continued to spend within the nest enclosure. Despite a reduction in food intake of approxi- mately $50 \%$ under the IFI 512 condition, average time spent in the nest decreased by only $11 \%$ (see Table 2 ). It may be that the nest time served a thermoregulatory function in the face of reduced food intake. However, given the large amount of baseline time spent in the nest it seems more likely that nest time was defended as an important commodity in itself. Researchers examining out-of-nest episodes in wild mouse species argue that forays from the nest are fraught with danger for the animal (Daan \& Slopsema, 1978; Madison, 1985). Therefore, they argue, each species has evolved a pattern and amount of out-of-nest time that minimizes the risk of predation relative to foraging costs and reproductive gains. Such a view would explain why the variation in nest time in the present study was so small, and why the animals frequently returned to the nest between food pellets at longer IFIs.

\section{The Classification of Adjunctive Behavior}

At a descriptive level, our results were generally in agreement with previous studies that divided adjunctive behavior into two broad classes on the basis of postprandial timing. However, the categories of behavior did not fit neatly into either Staddon's (1977) interim/facultative typology or Cohen et al.'s (1985) time-locked/ general activity typology. In particular, Staddon's typology failed to predict either the marked time-locked nature of many responses or which responses would show schedule-induced increases.

In partial support of Cohen et al.'s (1985) model, many of the responses that emerged immediately after feeding showed peak expression at about the same time across the different IFIs. However, these "time-locked" categories of behavior, for example, activity, wait by feeder, drinking, or rearing, did not appear to be any more stereotyped or repetitive than such responses as running or wait by water, which emerged later in the interval. Again in support of Cohen et al.'s model, some of the responses that peaked later in the IFIs also showed schedule-induced increases as the IFI was extended. However, this second set of responses did not appear to be limited to a "general activity" category. For example, retreating to the nest was a major component in this category.

The ordering of responses during the IFI was both more complex and more food-related than had previously been thought. For example, the "time-locked" set of responses that emerged soon after feeding included elements that could be characterized as focal search, that is, responses appropriate to the continued search of an immediate feeding site. These responses included head in feeder and wait by feeder (including episodes of sniffing along the floor and quiet waiting), responses that would be appropriate for locating or capturing additional food items in the area. At shorter delays (IFI 16 and 32), these responses largely dominated the interfood interval.

The time-locked character of these responses may be related to their role in postprandial focal search and subsequent transition to more expansive search. The data in 
Figure 5 suggest that the primary period for this postprandial search activity was limited to the first $32-64 \mathrm{sec}$ following food delivery. Other data (e.g., Killeen, 1975; Reid \& Dale, 1983; Reid \& Staddon, 1982) suggest that the duration of the postprandial arousal phase is not entirely "time-locked" but depends on several variables, including deprivation and the amount of food per access.

At longer intervals, the transition period involved more expansive search (e.g., movement away from the feeder, including rearing) along with postprandial drinking (see Figures 4 and 5). The midpoint of the longer intervals (IFI 128 to IFI 512) tended to include more general search activities (e.g., wheel-running and perhaps chewing) as well as some responses that appeared to be relegated to this period due to time constraints. These responses included retreat to the nest and wait by water (including postwater grooming). The latter category presumably increased as a result of the increase in drinking, but its distribution peaked later in the interval. Finally, the interval ended with the re-emergence of focal search behavior (head in feeder, wait by feeder) in anticipation of the next food item.

In summary, the present results argue that behavior between pellet deliveries consists of a complex set of responses that in many cases can be functionally related to foraging for food. These responses include foodhandling (including the "snatch and run" pattern), postprandial focal search (e.g., head in feeder and wait by feeder), postprandial drinking and more expansive search activities (e.g., rearing, running, activity, and sometimes chewing), and anticipatory focal search (e.g., head in feeder, wait by feeder).

\section{The Sources of Adjunctive Behavior}

The distribution of water-related responses (head in water, drinking, and wait by water) that emerged under periodic feeding conditions was closely related to the distributions of these responses around unconstrained feeding in baseline. But, although in baseline these responses emerged around discrete bouts of eating, under the periodic feeding schedule, these responses were recruited around each pellet. It was as though each pellet began and ended a feeding bout, and the rats showed the appropriate distribution and amount of pre- and postprandial behavior. In fact, the overall increases in the amount of water-related measures obtained under the periodic schedules could be accounted for solely on the basis of this increased opportunity for expression.

However, periodic food availability did more than simply reinstitute pre- and postprandial distributions of behavior. It produced a marked increase in anticipatory food-directed responses, such as head in feeder and wait by feeder. And this increase in anticipatory feeder-directed responses effectively competed with several other preprandial responses, resulting in a relative decrease in the frequency (per opportunity) of activity, nest, rear, and (for some animals) run. Similarly, though to a lesser extent, the distribution (per opportunity) of more explora- tory behavior (e.g., activity and wheel-running) increased above the baseline postprandial distribution.

In short, these data suggest that adjunctive behavior obtained in the present study emerged from three sources: (1) the treatment of individual pellets as though they were bouts of feeding, thereby markedly increasing the opportunity for normal pre- and postprandial behavior, (2) a marked increase in the preprandial behaviors specifically directed toward the site of expected food (focal search behaviors) immediately before pellet delivery, and (3) a smaller increase in immediate postpellet focal search and the distribution of more general exploratory behaviors potentially related to feeding. This view implies that the more appropriate baseline for assessing the excessiveness and changes in timing of adjunctive behavior is the behavioral distribution around unconstrained feeding; but, more importantly, it calls attention to the functional nature of adjunctive behavior and the multiple mechanisms that underlie it.

Some readers may object to our classification of adjunctive behavior into focal and extended search behavior on the grounds that these categories imply a function in obtaining food. In the present case, adjunctive behavior had no effect in producing food, because the site and timing of food delivery were fixed by the experimenter. However, we propose that adjunctive behavior is related to phylogenetically based linkages (in sequencing and timing) that would be effective in procuring food in more normal circumstances. In the absence of more effective contingencies, and to a lesser extent even in their presence, we suggest that these phylogenetic linkages will continue to be expressed, especially when learned feeding strategies do not lead to satiation.

Both the timing and amount of adjunctive drinking appeared highly related to the distributions of pre- and postprandial drinking in free-feeding baseline. Interestingly, the timing of the emergence of postprandial drinking places it near the point of transition between feederdirected behaviors and more expansive exploratory behavior. This timing suggests that the linkage between feeding and drinking occurs at the point at which the rat makes a transition from feeding at one site to a search for other sites or a transition to other activities.

Although a bias toward transitions to search for water was not functional in the current experimental context, a motivational link between feeding and drinking may be an appropriate search strategy in the rat's selection environment. Water is required for processing newly ingested food, and it should be more efficient for the rat to avoid depleting its internal water stores by obtaining water during the same out-of-nest activity period. Whatever the ultimate cause, a linkage between eating and drinking appears to be firmly established (Falk, 1971, 1977; Kissileff, 1969).

An argument based on foraging-based linkages can also be made for the pigeon's tendency to attack nearby conspecifics under periodic feeding schedules. These attacks are most likely to occur on the transition away from the 
feeder area to other parts of the chamber (Campagnoni et al., 1986; Yoburn, Cohen, \& Campagnoni, 1981). Such attacks appear to be a normal part of the pigeon's ground-foraging repertoire (Murton, 1965), and presumably serve to drive competitors away from potential nearby food. It seems likely that both excessive drinking and excessive attack may depend on ecologically functional links between these responses and feeding that become dysfunctional in the experimental context when their opportunity for expression is artificially increased.

One objection that is often raised against the conclusion that adjunctive drinking is related to pre- and postprandial drinking is that polydipsia usually takes a few days to develop in an experimental situation (Reynierse \& Spanier, 1968; Staddon \& Ayres, 1975). If polydipsia is simply the expression of normal food-associated drinking, then why doesn't it appear fully developed from the start? We think that there are two reasons that polydipsia is slow to develop. Recent data (Reid \& Dale, 1983; Reid \& Staddon, 1982) indicate that feeder-directed behavior (focal search) directly competes with drinking. Thus, if feeder-directed search showed a rapid increase when a periodic feeding schedule was imposed, drinking would have more competition initially and its emergence would be delayed.

Secondly, our data suggest that drinking emerges when the rat treats the postpellet time under the periodic schedule as if it were the end of a feeding bout, that is, the period of time after the rat has given up eating. However, in freefeeding baseline, rats typically eat $15-20$ pellets per feeding bout. Thus, under periodic schedules, the rat must first learn that each feeding opportunity is limited to a single pellet and that the period immediately following a food pellet is a time when further food is unavailable (see Reid \& Staddon, 1985; Staddon, 1977). It seems reasonable to assume that it should require a few days for the rat to adjust to such a periodic schedule before consistent patterns of postprandial drinking emerge.

This last point is particularly important because there is good evidence that feeding does not immediately elicit drinking. Reid and Staddon (1982; see also Reid \& Dale, 1983) have shown that the immediate effect of extra food is to reduce drinking, primarily by competition from fooddirected search. Our data suggest that food-associated drinking is most likely to emerge at the time of transition away from the feeder area after feeder-directed behavior has ended. These findings are consistent with the observation that schedule-induced drinking is likely to emerge in the context of feeding but in the presence of stimuli that signal the local absence of food (Staddon, 1977).

Recently, Reid and Staddon (1982) have argued that such an inverse relationship between feeding and drinking could be explained by a process of disinhibition. For example, in the present case, if both focal search and drinking were instigated by feeding but focal search were dominant and inhibited drinking, then a postprandial distribution of drinking could result from the disinhibition of drinking after focal search had been abandoned. Thus, stimuli that signal the absence of food may reduce focal search and indirectly increase drinking. Our results suggest that the closer to food such stimuli are presented, the more effective they should be in reducing focal search and promoting a transition to drinking.

In summary, our data imply that the adjunctive behavior that develops under periodic feeding schedules is not extraneous behavior but, rather, behavior related to feeding and foraging for food. As we have argued elsewhere (Timberlake, 1983; Timberlake \& Lucas, 1985, in press), the reliable emergence of functionally organized behavior under periodic schedules argues strongly for the presence of complex systems of behavior organized around important biological variables, such as feeding. The present study supports this view, and suggests that our concept of the "appetitive structure" of a feeding episode needs to be extended to include behavior that both precedes and follows feeding.

\section{REFERENCES}

Bolles, R. C., \& Moot, S. A. (1973). The rat's anticipation of two meals a day. Journal of Comparative \& Physiological Psychology, 83, 510-514.

Campagnoni, F. R., Lawler, C. P., \& Cohen, P. S. (1986). Temporal patterns of reinforcer-induced general activity and attack in pigeons. Physiology \& Behavior, 37, 577-582.

CoCHRAN, W. G. (1954). Some methods for strengthening the common chi-square tests. Biometrics, 10, 417-451.

Cohen, P. S., Looney, T. A., Campagnoni, F. R., \& Lawler, C. P. (1985). A two-state model of reinforcer-induced motivation. In F. R. Brush \& J. B. Overmier (Eds.), Affect, conditioning, and cognition: Essays on the determinants of behavior (pp. 281-297). New York: Erlbaum.

DAAN, S., \& SlopSema, S. (1978). Short-term rhythms in foraging behavior of the common vole, Microtus auralis. Journal of Comparative \& Physiological Psychology, 127, 215-227.

FAGAN, R. M., \& YounG, D. Y. (1978). Temporal patterns of behaviour: Durations, intervals, latencies, and sequences. In P. W. Colgan (Ed.), Quantitative ethology (pp. 79-114). New York: Wiley.

FALK, J. L. (1961). Production of polydipsia in normal rats by an intermittent food schedule. Science, 133, 195-196.

FALK, J. L. (1969). Conditions producing psychogenic polydipsia in animals. Annals of the New York Academy of Sciences, 9, 19-25.

FALK, J. L. (1971). The nature and determinants of adjunctive behavior. Physiology \& Behavior, 6, 577-588.

FALK, J. L. (1977). The origin and function of adjunctive behavior Animal Learning \& Behavior, 5, 325-335.

Hutchinson, R. R., Azrin, N. H., Hunt, G. M. (1968). Attack produced by intermittent reinforcement of a concurrent operant response. Journal of the Experimental Analysis of Behavior, 11, 485-495.

KILleEN, P. (1975). On the temporal control of behavior. Psychological Review, 82, 89-115.

KING, G. D. (1974). Wheel running in the rat induced by a fixed-time presentation of water. Animal Learning \& Behavior, 2, 325-328.

KISSILEFF, H. R. (1969). Food-associated drinking in the rat. Journal of Comparative \& Physiological Psychology, 67, 284-300.

Lang, W. J., Latiff, A. A., McQueen, A., \& Singer, G. (1977). Self-administration of nicotine with and without a food delivery schedule. Pharmacology, Biochemistry \& Behavior, 7, 65-70.

LEHNER, P. N. (1979). Handbook of ethological methods. New York: Garland Press.

LeVITSKY, D., \& ColLIER, G. (1968). Schedule-induced wheel running. Physiology \& Behavior, 3, 571-573.

MAdison, D. M. (1985). Activity rhythms and spacing. In R. H. Tamarin (Ed.), Biology of new world microtines (pp. 373-419). Shippensburg, PA: American Society of Mammologists.

MCFARLAND, D. J. (1974). Time-sharing as a behavioral phenomenon. 
In D. S. Lehrman, J. S. Rosenblatt, R. A. Hinde, \& E. Shaw (Eds.), Advances in the study of behavior (Vol. 4, pp. 201-225). New York: Academic Press.

McFarland, D. J., \& LLOYD, I. (1973). Time-shared feeding and drinking. Quarterly Journal of Experimental Psychology, 25, 48-61.

MELCER, T., \& TIMBERLAKE, W. (1986). Running and drinking by rats outside the schedule session. Behavioural Processes, 13, 29-38.

Mendelson, J., \& ChillaG, D. (1970). Schedule-induced air licking in rats. Physiology \& Behavior, 5, 535-537.

Murton, R. K. (1965). The Wood-pigeon. London: Collins.

OEI, T. P. S., \& SinGER, G. (1979). Effects of a fixed-time schedule and body weight on ethanol self-administration. Pharmacology, Biochemistry \& Behavior, 10, 767-770.

Peterson, M. R., \& Lyon, D. O. (1978). Schedule-induced polydipsia in rats living in an operant environment. Joumal of the Experimental Analysis of Behavior, 29, 493-503.

ReID, A. K., \& DALE, R. H. I. (1983). The dynamic effects of food magnitude on interim-terminal interaction. Joumal of the Experimental Analysis of Behavior, 39, 135-148.

REID, A. K., \& StadDon, J. E. R. (1982). Schedule-induced drinking: Elicitation, anticipation, or behavioral interaction? Journal of the Experimental Analysis of Behavior, 38, 1-18.

ReID, A. K., \& Staddon, J. E. R. (1985). Mechanisms of schedule entrainment. In S. J. Cooper \& C. T. Dourish (Eds.), Neurobiology of behavioral stereotypy. Oxford: Oxford University Press.

REYNIERSE, J. H., \& SPANIER, D. (1968). Excessive drinking in rats' adaptation to the schedule of feeding. Psychonomic Science, 10, 95-96.

ROPER, T. J. (1978). Diversity and substitutibility of adjunctive activities under fixed-interval schedules of food reinforcement. Journal of the Experimental Analysis of Behavior, 30, 83-96.

ROPER, T. J. (1980). Behaviour of rats during self-initiated pauses in feeding and drinking, and during periodic response-independent delivery of food and water. Quarterly Journal of Experimental Psychology, 32, 459-472.

ROPER, T. J. (1981). What is meant by the term "schedule-induced" and how general is schedule induction? Animal Learning \& Behavior, 9, 433-440.

Roper, T. J., \& Crossland, G. (1982). Schedule-induced wood-chewing in rats and its dependence on body weight. Animal Learning \& Behavior, 10, 65-71.

ROPER, T. J., \& NiETo, J. (1979). Schedule-induced drinking and other behavior in the rat, as a function of body weight deficit. Physiology \& Behavior, 23, 673-678.

StadDON, J. E. R. (1977). Schedule-induced behavior. In W. K. Honig \& J. E. R. Staddon (Eds.), Handbook of operant behavior (pp. 125-152). New York: Prentice-Hall.
Staddon, J. E. R., \& AYREs, S. L. (1975). Sequential and temporal properties of behavior induced by a schedule of periodic food delivery. Behaviour, 54, 26-49.

Staddon, J. E. R., \& Simmelhag, V. (1971). The "superstition" experiment: A reexamination of its implications for the principles of adaptive behavior. Psychological Review, 78, 3-43.

Takahashi, R. N., Singer, G., \& OEI, T. P. S. (1978). Scheduleinduced self-injection of d-amphetamine by naive animals. Pharmacology, Biochemistry \& Behavior, 9, 857-861.

TimberLAKE, W. (1982). Controls and schedule-induced behavior. Animal Learning \& Behavior, 10, 535-536.

TimberLAKE, W. (1983). The functional organization of appetitive behavior: Behavior systems and learning. In M. D. Zeiler \& P. Harzem (Eds.), Advances in the analysis of behavior: Vol. 3. Biological factors in learning (pp. 177-221). Chichester, England: Wiley.

Timberlake, W., \& LuCAs, G. A. (1985). The basis of superstitious behavior: Chance contingency, stimulus substitution, or appetitive behavior. Joumal of the Experimental Analysis of Behavior, 44, 279-299.

Timberlake, W., \& LuCAS, G. A. (in press). Behavior systems and learning: From misbehavior to general principles. In S. B. Klein \& R. R. Mowrer (Eds.), Contemporary learning theories. Hillsdale, NJ: Erlbaum.

Wallace, M., \& Singer, G. (1976). Adjunctive behavior and smoking induced by a maze solving schedule in humans. Physiology \& Behavior, 17, 849-852.

Wetherington, C. L., Brownstein, A. J., \& Shull, R. L. (1977). Schedule-induced nunning and chamber size. Psychological Record, 27, 703-713.

YoBURN, B. C., \& CoHEN, P. S. (1979). Assessment of attack and drinking in White King pigeons on response-independent food schedules. Journal of the Experimental Analysis of Behavior, 31, 91-101.

Yoburn, B. C., Cohen, P. S., \& Campagnoni, F. R. (1981). The role of intermittent food in the induction of attack. Journal of the Experimental Analysis of Behavior, 35, 101-117.

\section{NOTE}

1. Following Kissileff (1969), the terms preprandial and postprandial are used here to describe behavior that normally occurs before and after periods of feeding. As will be argued later, these categories may include behavior that occurs in anticipation of feeding or following the offset of feeding. However, the categories do not imply that the behavior is directly elicited by food.

(Manuscript received December 8, 1986; revision accepted for publication June 11,1987 .) 